Short Report

\title{
Difference of Subtypes of Hepatitis B Surface Antigen between Asymptomatic Carriers and Patients with Chronic Liver Diseases
}

\author{
Shozo Nakamura, Yoshiaki Takezawa* and \\ Toshiyuki Maeda $\uparrow$ \\ Tohoku University Health Center, Sendai 980, *the First \\ Department of Internal Medicine, Tohoku University School \\ of Medicine, Sendai 980, and $\dagger$ Hikarigaoka Spellman \\ Hospital, Sendai 983
}

\begin{abstract}
Nakamora, S., Takezawa, Y. and MaEda, T. Difference of Subtypes of Hepatitis $B$ Surface Antigen between Asymptomatic Carriers and Patients with Chronic Liver Diseases. Tohoku J. exp. Med., 1980, 132 (3), 367-368Subtypes of hepatitis B surface antigen $(\mathrm{HBsAg})$ were studied in 20 asymptomatic $\mathrm{HBs} A \mathrm{~g}$ carriers and $18 \mathrm{HBsAg}$-seropositive patients with chronic hepatitis or liver cirrhosis. Among them 25 were university students. In the male university students, subtype adw was found to be significantly higher in asymptomatic HBsAg carriers than in patients with chronic hepatitis $(p=0.04)$. The difference in subtypes of HBsAg between male asymptomatic carriers and male patients with liver disease was further confirmed by the combined results of university students and other subjects $(p=0.007)$. - subtypes of hepatitis B surface antigen; asymptomatic hepatitis $\mathrm{B}$ surface antigen carrier; chronic hepatitis; liver cirrhosis; university students
\end{abstract}

In Japan the view is widely held currently that chronic hepatitis B develops in adolescence mostly from asymptomatic hepatitis B surface antigen ( $\mathrm{HBsAg}$ ) carrier state (Suzuki 1977). Difference of subtypes of HBsAg between asymptomatic HBsAg carriers and patients with chronic hepatitis or liver cirrhosis has not been noted, though the difference of subtypes between patients with acute hepatitis B and asymptomatic HBsAg carriers was pointed out (Kojima et al. 1975). We recently found a significant difference of subtypes of HBsAg between asymptomatic HBsAg carriers and patients with chronic liver diseases in university students. This was confirmed by further studies in other carriers and patients visiting the hospitals. The results will be described in the present report.

Subtypes of HBsAg were studied in 20 asymptomatic $\mathrm{HBsAg}$ carriers and in $18 \mathrm{HBsAg}$ seropositive patients with chronic hepatitis or liver cirrhosis. Among them 19 asymptomatic carriers and 6 patients with chronic hepatitis were university students. HBsAg was determined by the reversed passive hemagglutination method (Germain et al. 1973), and subtypes of HBsAg by the passive hemagglutination inhibition method (Imai et al. 1974). Statistical significance was tested by Fischer's direct calculation method of probability.

In the male university students, $57 \%$ of the asymptomatic carriers had subtype adw and $43 \%$ adr (Table 1), while all the male patients with chronic hepatitis $B$ had subtype $a d r$. A significant difference was found between male asymptomatic carriers and male patients with chronic hepatitis B $(p=0.04)$.

The combined results of university students and other subjects are shown in Table 2. Among the male asymptomatic carriers, $60 \%$ had subtype $a d w$ and $40 \% a d r$, while $8 \%$ of the seropositive male patients with chronic hepatitis or liver cirrhosis showed subtype adw and $92 \% a d r$. The difference between male asymptomatic carriers and patients with liver

Received for publication, May 23, 1980. 
TaBLe 1. Subtypes of HBs.Ag in university students

\begin{tabular}{cccc} 
Sex & \multicolumn{1}{c}{ Diagnosis } & $a d w$ & \multicolumn{1}{c}{$a d r$} \\
M & Asymptomatic carrier & $8(57 \%)^{*}$ & $6(43 \%)^{*}$ \\
M & Chronic hepatitis & $0(0 \%)^{*}$ & $5(100 \%)^{*}$ \\
F & Asymptomatic carrier & $2(40 \%)$ & $3(60 \%)$ \\
$\mathrm{F}$ & Chronic hepatitis & 1 & 0 \\
$\mathrm{M}+\mathrm{F}$ & Asymptomatic carrier & $10(53 \%)$ & $9(47 \%)$ \\
$\mathrm{M}+\mathrm{F}$ & Chronic hepatitis & $1(17 \%)$ & $5(83 \%)$ \\
\hline & * The difference between male asymptomatic carriers \\
and male patients with chronic hepatitis is statistically sig- &
\end{tabular}

TABLe 2. Subtypes of $H B s A g$ in university students and other subjects

\begin{tabular}{clrr} 
Sex & \multicolumn{1}{c}{ Diagnosis } & \multicolumn{1}{c}{$a d w$} & $a d r$ \\
\cline { 3 - 4 } M & Asymptomatic carrier & $9(60 \%)$ & $6(40 \%)$ \\
M & Chronic hepatitis or cirrhosis & $1(8 \%)^{*}$ & $11(92 \%)^{*}$ \\
F & Asymptomatic carrier & $2(40 \%)$ & $3(60 \%)$ \\
F & Chronic hepatitis or cirrhosis & $4(67 \%)$ & $2(339)$ \\
M+F & Asymptomatic carrier & $11(55 \%)$ & $9(45 \%)$ \\
M+F & Chronic hepatitis or cirrhosis & $5(28 \%)$ & $13(729)$
\end{tabular}

* The differences from male asymptomatic carriers and from female patients with chronic hepatitis or liver cirrhosis are both statistically significant $(p=0.007$ and $p=0.02)$.

diseases was statistically significant $(p=0.007)$. In females such a tendency was not observed. In the female patients with chronic liver diseases, $67 \%$ had subtype adw and $33 \% a d r$, showing a significant difference from the male patients with chronic liver diseases $(p=0.02)$.

In Japan south to north gradient of $r$ and reciprocal gradient of $w$ in asymptomatic carriers was noted (Yamashita et al. 1975). Geographical difference of subtypes of HBsAg in chronic liver diseases, however, was not evident; the adw: adr ratio in asymptomatic carriers in Gifu, Tokyo, and Niigata was $1: 9,3: 7$, and 4:6, respectively, while the ratio in chronic hepatitis and liver cirrhosis in these areas was all about 2:8 (Kojima et al. 1975; Oda et al. 1975). It may not be fortuitous that significant difference of subtypes of $\mathrm{HBsAg}$ between male asymptomatic carriers and male patients with chronic liver diseases was shown in the northern city, Sendai.

\section{Acknowledgment}

We wish to thank Dr. F. Tsuda and Dr. M. Mayumi, Hepatitis Division of Tokyo Metropolitan Institute of Medical Science, for the determination of subtypes of $\mathrm{HBsAg}$.

\section{References}

1) Germain, K.H., Sturdivant, S.K. \& Rightsel, W.A. (1973) Evaluation of a red cell agglutination test for detection of Australia antigen (HBAg). Appl. Microbiol., 25, 524-527.

2) Imai, M., Yamashita, Y., Miyakawa, Y. \& Mayumi, M. (1974) Hemagglutination inhibition assay of the common determinants and subspecificities of Australia antigen. Immunology, 27, 871-878.

3) Kojima, M., Kametani, M. \& Fukuta, N. (1975) Subtypes of hepatitis B surface antigen and epidemiology. Sogo Rinsho, 24, 2346-2453. (Japanese)

4) Oda, T., Suzuki, H. \& Mitamura, K. (1975) Clinical study of Australia antigen. $J$. Jap. med. Ass., 73, 182-190. (Japanese)

5) Suzuki, H. (1977) Hepatitis B. Rinsho Seijinbyo, 7, 13-19. (Japanese)

6) Yamashita, Y., Kurashina, S., Miyakawa, Y. \& Mayumi, M. (1975) South-to-north gradient in distribution of the determinant of hepatitis $\mathrm{B}$ surface antigen in Japan. J. Infect. Wis., 131, 567-569. 